\title{
Examination of a Sigmoid Shape Composition for BMI Fluctuation and Fat Percentage
}

\author{
Katsunori Fujii ${ }^{1, ~ *, ~ N o z o m i ~ T a n a k a ~}{ }^{2}$, Yuki Takeyama ${ }^{1}$, Tohru Ishigaki ${ }^{3}$ \\ ${ }^{1}$ Graduate School of Business Administration and Computer Science, Aichi Institute of Technology, Toyota, Japan \\ ${ }^{2}$ Department of Sport and Health Science, Tokai Gakuen University, Miyoshi, Japan \\ ${ }^{3}$ Department of Exercise and Health Science, Aichi University of the Arts, Nagakute, Japan
}

\section{Email address:}

fujii@aitech.ac.jp (K. Fujii), tanaka-n@tokaigakuen-u.ac.jp (N. Tanaka), yuki319takeyama@yahoo.co.jp (Y. Takeyama), isshy@mail.aichi-fam-u.ac.jp (T. Ishigaki)

${ }^{*}$ Corresponding author

\section{To cite this article:}

Katsunori Fujii, Nozomi Tanaka, Yuki Takeyama, Tohru Ishigaki. Examination of a Sigmoid Shape Composition for BMI Fluctuation and Fat Percentage. American Journal of Sports Science. Vol. 9, No. 3, 2021, pp. 60-65. doi: 10.11648/j.ajss.20210903.11

Received: June 25, 2021; Accepted: July 12, 2021; Published: July 16, 2021

\begin{abstract}
Body mass index (BMI) is an index of physique proposed by Quetelet, and has been given meaning as the Quetelet function, which is the ratio of body weight to the square of height. In recent years, BMI has been considered an indicator of obesity due to its high correlation with body fat percentage. However, while the relationship between BMI and body fat percentage shows a high correlation, the composition of that relationship is not well understood. Moreover, there is almost no information on the relational composition in males and females. In this study, we investigated that relational composition to determine whether the relationship between BMI and body fat percentage follows a linear trend or a curvilinear trend. For that purpose, we analyzed the changes in body fat percentage associated with fluctuations in BMI. For BMI fluctuations, the body fat percentage of the scale width of one BMI unit was calculated when BMI was increased one unit at a time, and the changes in the calculated statistics were analyzed. The type of function series to which the relationship between BMI and body fat percentage fits was then verified. The results demonstrated that in males the fluctuations in body fat percentage with respect to BMI show a sigmoid shape. In males, when BMI exceeds 25 , the velocity of body fat percentage decreases and soon reaches a constant level. A sigmoid curve is thought to be a finding that means a critical point in the inflection point phenomenon. Therefore, the fact that the change in body fat percentage with respect to BMI shows a sigmoid shape means that, in males, body fat percentage shows a critical point at a BMI of 25. In females, however, the critical point is a BMI of 28.6 and body fat percentage reaches a constant level when a point higher than that in males is exceeded, but the shape could not be judged to be sigmoid. There are limits to BMI for humans, we propose a limiting body fat percentage hypothesis in which body fat percentage becomes constant from a BMI of around 32.
\end{abstract}

Keywords: BMI, Body Fat Percentage, Fluctuation, Sigmoid Shape

\section{Introduction}

A sigmoid shape is derived from the Greek letter sigma $(\sigma)$, and is said to mean a line similar to a sigma. In other words, it is taken to mean an S-shaped curve like a logistic curve. Sigmoid curves have been used as typical of the general curve in Scammon's growth curves. Height growth in particular belongs to the general shape, and in a classic study on height growth [1], the finding was presented that a logistic curve fit a method describing a sigmoid curve as the general pattern. Following from this research, the relational composition between height growth and a sigmoid shape was taken up. First, a general logistic function showing a sigmoid was used, after which double logistic, triple logistic, and multiple logistic functions as well as logistic system functions have been used as methods to describe height growth [2-5]. Recently, however, Fujii [6] proposed the Fujimmon's growth curves and said that the general growth pattern is not necessarily a sigmoid shape. Then, using the wavelet interpolation model, he proposed a fractal pattern to describe functions, from which height growth was found to be distinct from a sigmoid shape. 
From the beginning, there has been no really good evidence for the relational composition between the general growth pattern of height and a sigmoid shape. Among Scammon's growth curves, the general growth pattern was simply judged to show an $\mathrm{S}$ shape in observations with the naked eye. In fact, the growth pattern for height has a relationship that is far from a sigmoid shape. Rather, as seen in the statement of Marubini [2] that the Gompertz function more accurately describes the height growth pattern, a logistic function (sigmoid) curve has low accuracy in describing the shape. The causal relationship between a sigmoid shape and growth curves is thought to be weak. However, a sigmoid shaped curve is simply a curve that resembles the Greek letter sigma $(\sigma)$, and nowhere is it prescribed. Since a logistic function shows an S-shaped curve, there are grounds for fitting it to a sigmoid curve. Therefore, a logistic curve is a sigmoid curve.

Another function includes polynomials, which are third-order polynomials. With third-order polynomials, the differential is second order and they show exactly the same sigmoid curve as a logistic function. Thus, sigmoid curves can be specified as functions in which the differential shows a convex shape. However, logistic functions and polynomials simply fit sigmoid curves; this does not mean they cover all sigmoid shapes. Assuming that a given relational composition has a sigmoid shape, can that relational composition be explained with a logistic function or a polynomial? If the relational function can be described with a logistic function or a polynomial, it then cannot explain a sigmoid shape composition. This exposes the limitations of specified functions in prescribing sigmoid shapes. Considered in this way, what is needed is a method that can describe relational compositions but is not based on specific functions. The wavelet interpolation model proposed by Fujii [7] and Fujii et al. [8] has the characteristic of being able to describe polynomials and logistic functions themselves as they are. That is, they have the characteristic of being able to describe curves assuming a given positional composition.

The relational composition between body mass index (BMI) and body fat percentage is a mysterious one. BMI was originally an index of physique devised by Quetelet [9]. The positioning of BMI as an indicator of obesity in recent years began with the papers of Key et al [10] and Garrow and Webster [11], which have a meaning different from that intended by Quetelet [9]. This is because of the close relationship shown between BMI and body fat percentage. Later research showed that the meaning of BMI with respect to obesity differs between men and women. Wang and Bachrach [12] reported that the correlation between BMI and body fat percentage was $r=0.72$ in women but a low $r=0.15$ in men, and that BMI is more strongly affected by lean body mass than by body fat mass. Maynard et al [13] also reported that the increases in BMI in the growth period depend greatly on changes in lean body mass. With data from Japanese people, Tahara et al (2002) [14] reported that a remarkable increase in lean body mass is seen together with growth in males.

Tanaka et al. [15-17] composed a regression polynomial of body fat percentage against BMI in Japanese and Korean junior high school students, and from the derived evaluation chart verified the composition of change in the degree of fat accumulation with respect to BMI. They then investigated physical strength based on those findings, and demonstrated that body fat has a negative effect on physical strength even in people not judged to be obese. In their findings, the correlation between BMI and body fat percentage was around $r=0.7$ in boys. The meaning of BMI as an index of fatness is thought to have been established from findings such as these. In the studies of Key et al [10] and Garrow and Webster [11], a linear regression line was derived for body fat percentage against BMI. In the reports of Tanaka et al. [15-17], a third-order regression polynomial was derived for body fat percentage against BMI. Thus, in the relational composition between BMI and body fat percentage, the sigmoid shape of a third-order polynomial can be assumed.

In the findings of Tanaka et al. [15-17], a third order polynomial was derived from the application of a least squares approximation polynomial to the relational composition between BMI and body fat percentage. It became clear as a result that this does not reach the level of specifying that a sigmoid shape can describe a cubic polynomial. However, it is possible to assume a sigmoid shape. Thereupon, to examine the relational composition between BMI and body fat percentage in detail, we ascertained the changes in body fat percentage with fluctuations in BMI. For BMI fluctuations, body fat percentage was calculated with the scale width of one unit when BMI was raised one unit at a time. By analyzing those changes, we examine whether the relational composition between BMI and body fat percentage formed a sigmoid shape. Then, continuing along this same line, we discuss the hypothesis that body fat percentage shows an upper limit at a given BMI.

\section{Methods}

\subsection{Subjects}

Using the data for 15,938 male students and 5,646 female students at one university, we first excluded those with BMI of $\leq 14$ or $\geq 32$. The remaining 15,714 males and 5,625 females were the subjects. BMI was the value obtained by dividing body weight $(\mathrm{kg})$ by the square of height $(\mathrm{m})$.

\subsection{Measurements of Body Composition}

Height was measured in units of $1 \mathrm{~mm}$ using a stadiometer. Weight and body composition were measured with a body composition monitor (RD-503, Tanita) that uses dual-frequency bioelectrical impedance analysis (BIA). Among the BIA monitors that are the most widely used in the world, multifrequency BIA is reported to have higher estimation accuracies for body water and body fat percentage than body fluid or DEXA methods [18]. The monitor used had two electrodes on either side of the scale, and subjects stood on the electrodes in the scale in bare feet. From the measurements, lean body mass can be estimated separately for body water content, protein content, and estimated bone 
(mineral) content, and body fat percentage is calculated together with body weight. With this device, body weight and protein content were estimated in units of $100 \mathrm{~g}$, and estimated bone mass was estimated in units of $10 \mathrm{~g}$. When estimating body composition (body water content) with BIA, a conference investigating BIA standardization agreements held in Rome in 1993 concluded that it is preferable to have the subject in a supine position with four electrodes attached to the right arm and leg. In this study, however, measurements were made with subjects in a standing posture, which is in widespread general use, including for general consumer uses, for the purpose of inexpensive and simple measurements of body composition.

\subsection{Analysis}

\subsubsection{Analytical Procedures}

BMI was separated into single units, and the statistics for body fat percentage included in those scale bands were calculated. Data were then smoothed by applying the least squares approximation polynomial of BMI against the mean value of calculated body fat percentage. The wavelet interpolation model was also applied to the numerical values to which the least squares approximation polynomial had been applied, and a velocity curve was derived with differentiation of the distance curve.

\subsubsection{Application of the Wavelet Interpolation Model}

The wavelet interpolation method (WIM) is a technique to approximately describe true growth curves from given growth data. This is done by interpolating individual data with a wavelet function, drawing growth distance curves, and identifying the age at maximum peak velocity (MPV) from the growth velocity curve obtained with differentiation of the drawn distance curves.

\section{Results}

Table 1 shows statistics for the changes in body fat percentage with fluctuations in BMI. A tendency is shown for body fat percentage to rise together with increases in BMI. A least squares approximation polynomial of body fat percentage against BMI was applied to clarify this trend, as a result of which approximations with third-order polynomials were judged to be valid for males (Figure 1) and fifth order polynomials for females (Figure 2). Thus, an assumed sigmoid curve was shown for males, but for females the trend for increased body fat percentage with change in BMI continued and the upper limit for body fat percentage was ambiguous, which is unlike a sigmoid curve. Whether or not the shape was a sigmoid curve was then investigated by showing the rate of change in the distance value for body fat percentage together with changes in BMI. In this analysis, by applying the wavelet interpolation model and treating BMI changes as a time series, the distance curve of body fat percentage was described. Furthermore, an attempt was made to derive grounds for a sigmoid curve from the behavior of the velocity curve as a differential of that distance curve. Figures 3 and 4 are graphs with the application of the wavelet interpolation model to the distance values of body fat percentage with BMI fluctuations taken as a time series on the horizontal axis.

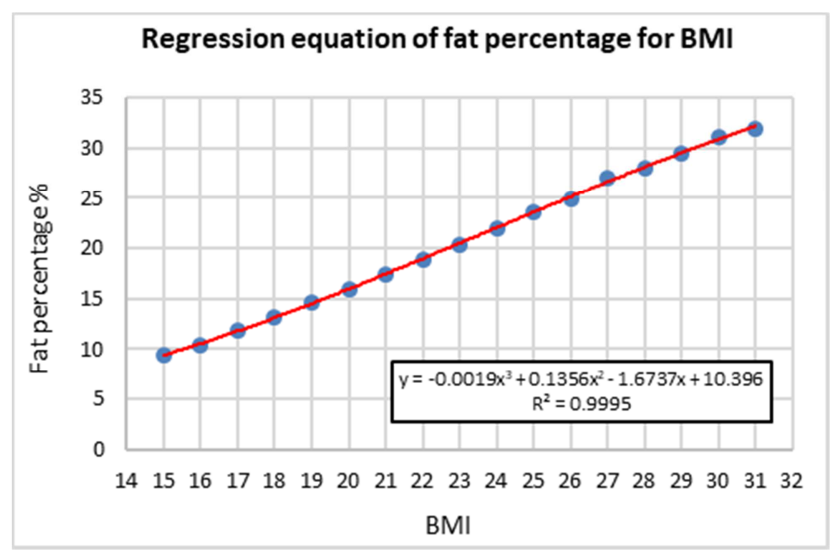

Figure 1. Regression equation of fat percentage for BMI in male.

Table 1. Statistics of fat percentage based on change of BMI.

\begin{tabular}{lllllll}
\hline \multirow{2}{*}{ BMI } & Male & \multicolumn{5}{c}{ Female } \\
\cline { 2 - 7 } & $\mathbf{n}$ & Mean of fat percentage & SD of fat percentage & $\mathbf{n}$ & Mean of fat percentage & SD of fat percentage \\
\hline 15 & 145 & 9.33 & 1.82 & 35 & 15.81 & 2.04 \\
16 & 541 & 10.42 & 1.95 & 203 & 17.66 & 1.69 \\
17 & 1232 & 11.87 & 2.12 & 447 & 19.58 & 1.83 \\
18 & 2027 & 13.19 & 2.33 & 777 & 21.62 & 2.28 \\
19 & 2424 & 14.65 & 2.54 & 1066 & 23.55 & 2.21 \\
20 & 2386 & 15.96 & 2.75 & 964 & 25.38 & 2.49 \\
21 & 1962 & 17.42 & 2.92 & 741 & 27.02 & 3.27 \\
22 & 1488 & 18.85 & 3.17 & 525 & 28.80 & 3.75 \\
23 & 1125 & 20.27 & 3.60 & 319 & 30.53 & 4.16 \\
24 & 778 & 21.95 & 3.32 & 184 & 31.79 & 3.93 \\
25 & 485 & 23.65 & 3.81 & 122 & 33.62 & 3.76 \\
26 & 344 & 24.94 & 3.80 & 79 & 35.23 & 4.71 \\
27 & 241 & 27.00 & 3.78 & 71 & 36.56 & 4.41 \\
28 & 177 & 28.02 & 3.81 & 37 & 39.63 & 5.67 \\
29 & 156 & 29.42 & 3.74 & 15 & 41.23 & 4.23 \\
30 & 111 & 31.12 & 4.34 & 21 & 42.46 & 43.03 \\
31 & 92 & 31.97 & 5.32 & 21 & \\
\hline
\end{tabular}




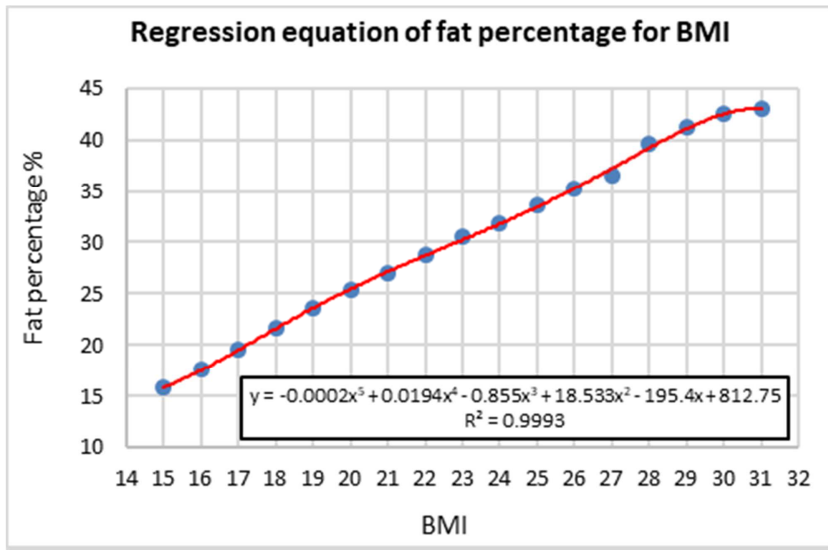

Figure 2. Regression equation of fat percentage for BMI in female.

Looking at the behavior of the velocity curve for males, the peak velocity is shown at a BMI of 25 . Afterward, the velocity gradually decreases. Hence, showing a single peak in the velocity curve is the basis for a sigmoid curve. However, for females BMI was 28.4 at the velocity peak, and the increases in body fat percentage accompanying BMI fluctuations tended to continue up to a high BMI value, which was a trend different from that in males. Thus, while a close relationship was shown between BMI and body fat percentage in females, body fat percentage did not increase from BMI that showed a certain upper limit.

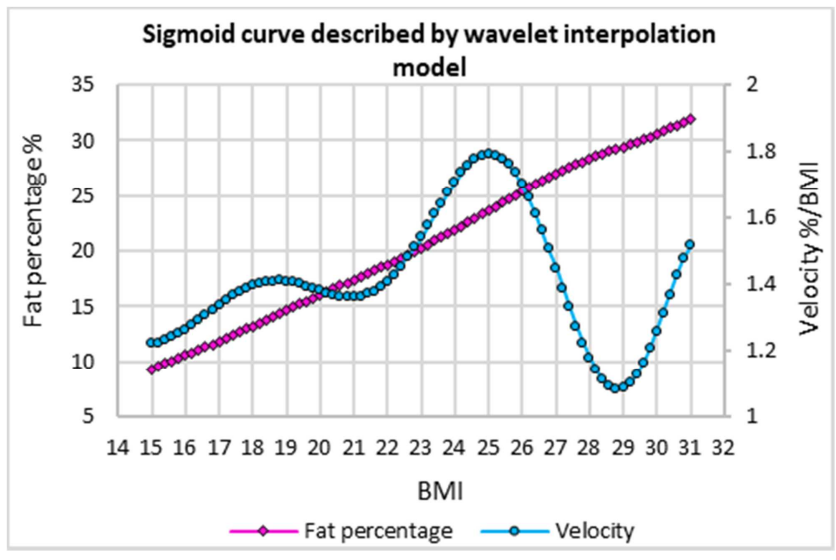

Figure 3. Sigmoid curve described by wavelet interpolation model in male.

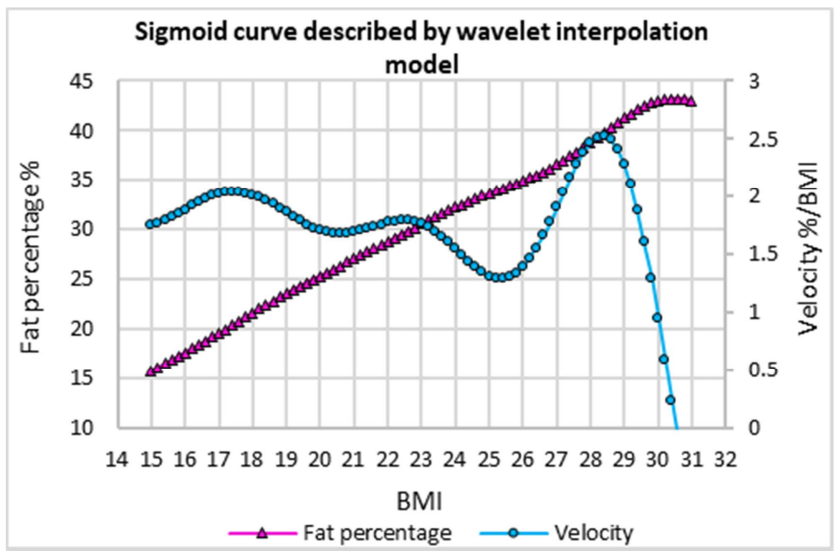

Figure 4. Sigmoid curve described by wavelet interpolation model in female.

\section{Discussion}

Quetelet [9] measured the physiques of 6,000 Scottish soldiers and 10,000 French volunteer soldiers, and derived the rule that the average adult body weight is proportional to the square of height. This is the Quetelet index, or BMI. Kuroki [19] said that the first to adopt BMI in Japan was in fact the pre-World War 2 Japanese army, not the medical profession. Of course, that was a time before the papers of Key et al [10] and Garrow and Webster [11] had come out. Yet while BMI can be calculated easily, it is not clear what grounds there are for it being an index of body fatness. Kuroki [19], generalizing from forest ecology that the ease of cutting down a tree is related to its height and diameter, derived the following BMI calculation with humans also taken to be cylindrical. He said it seemed to be proportional to the thickness of the human body.

$$
\mathrm{BMI}=\frac{\text { weight }(\mathrm{kg})}{\text { height }(\mathrm{m})^{2}}
$$

$$
\begin{gathered}
\frac{\text { weight }(\mathrm{kg})}{\text { height }(\mathrm{m})^{2}} \infty \frac{\text { height } \times \text { cross sectional area }}{\text { height } \times \text { height }} \\
\infty \frac{\text { height } \times A C(\text { abdominal circumference }) \times A C}{\text { height } \times \text { height }}=\frac{(A C)^{2}}{\text { height }}
\end{gathered}
$$

Hence, Kuroki [19], judging from the above formula, reported that the correlation between abdominal circumference and BMI was $r=0.9$ regardless of sex differences. However, he did not discuss the background for that in detail, and so did not present clear findings on the correlation between BMI and abdominal circumference.

In this study, we thought it may be possible to derive grounds for BMI as an index of obesity and fatness from its relationship with body fat percentage. In previous studies, regression analyses of body fat percentage with respect to BMI were reported to show the effectiveness of regression polynomials with very high correlations $[15,20]$. In particular, Fujii et al. [21] and Tanaka et al. [15, 17] advocated second or third order polynomial regression analyses from polynomial regression analyses of body fat percentage against BMI. If the regression polynomial of body fat percentage to BMI is third order, a sigmoid curve can be assumed. However, no sex difference is shown in regression polynomials. In this study, the idea of BMI fluctuations was devised anew, and the changes in body fat percentage with fluctuations in BMI was analyzed. With this, a difference between males and females becomes clear. In males, the results of applying a least squares regression polynomial to the changes in body fat percentage that accompany BMI fluctuations showed a gradual increasing trend in body fat percentage. Third order polynomials were judged to be valid, and a sigmoid curve was assumed. For females, unlike males, the gradual increasing trend in body fat percentage that accompany BMI fluctuations continued, and it was difficult to assume a sigmoid curve.

BMI was then set as a time series on the horizontal axis, 
and the wavelet interpolation model was applied to the distance changes in body fat percentage. Looking at the velocity curve in which the distance curve of body fat percentage was differentiated, the peak velocity in males was at a BMI of 25. This behavior is evidence that the changes in body fat percentage with respect to BMI show a sigmoid curve. In other words, from the fact that the changes in body fat percentage against BMI have a sigmoid shape, it is taken that body fat percentage enters a decreasing phase when BMI exceeds 25 , and that body fat percentage reaches an upper limit at a BMI of around 30-32. In females, body fat percentage velocity shows a peak at BMI of 28.4, and shows a behavior that differs from the sigmoid shape of males. However, it was demonstrated that in both males and females body fat percentage shows a constant amount at a BMI of around 32. Consequently, this phenomenon is thought to be a finding that suggests a critical point in the fluctuations of both fat percentage with respect to BMI.

The meaning of a sigmoid curve is that the S-shaped curve of logistic curves and the Greek letter sigma $(\sigma)$ are connected. At first this was given no special meaning, but because it was cited in the general shape of Scammon's [22] growth curves, sigmoid shapes have been applied in the background of the mathematical functions applied to human growth curves. In particular, in studies that have applied logistic functions to height growth based on classic studies, the meaning of inflection points in logistic curves that show sigmoid shapes is that they indicate the pubertal peak age. In recent findings, the basis for the sigmoid shape related to classification of the general growth pattern was verified from the wavelet interpolation model, leading to the Fujimmon's growth curves [6] proposed by Fujii [8]. Given this background, a sigmoid curve is thought to be a finding that means a critical point in the inflection point phenomenon. Therefore, the fact that the change in body fat percentage with respect to BMI shows a sigmoid shape means that, in males, body fat percentage shows a critical point at a BMI of 25 . That is, when BMI becomes larger than 25 the velocity of body fat percentage slows and then becomes constant. The critical point in females is at a BMI of 28.6, and body fat percentage becomes constant when a point that is higher than that in males is passed.

Height no longer increases once a person becomes an adult, but weight will continue to increase if consumed calories increase. Consequently, BMI also continues to increase. Of course, there are weight limits as humans, and it may be possible to propose a limiting body fat percentage hypothesis where body fat percentage becomes constant from a BMI of around 32. In the future it may be necessary to search for a critical point where the body fat percentage is constant for BMI, and to verify the limiting body fat percentage hypothesis with respect to BMI.

\section{Conclusion}

In this study we discussed the derivation of a basis for BMI as an index of obesity and fatness from the relationship with body fat percentage. For that purpose, we analyzed the changes in body fat percentage that occur with fluctuations in BMI for the relational composition of BMI and body fat percentage. Body fat percentage of the scale width of one unit of change in BMI was calculated, and the changes in the calculated statistics were analyzed. From this we examined whether the relational composition of BMI and body fat percentage could be a sigmoid shape. The results demonstrated that in males the fluctuation in body fat percentage with respect to BMI shows a sigmoid shape. For males, body fat percentage showed a critical point at a BMI of 25 , and when BMI exceeds 25 the velocity of body fat percentage decreases and soon becomes constant. However, in females body fat percentage becomes constant when a BMI of 28.6 is passed, a point higher than in males, but the shape could not be judged to be sigmoid. There are limits to BMI for humans, but it would seem to be possible to propose a limiting body fat percentage hypothesis where body fat percentage becomes constant from a BMI of around 32. In the future, it may be necessary to verify a limiting body fat percentage hypothesis with respect to BMI.

\section{References}

[1] Deming, J. (1957). Application of the Gompertz curve to the observed pattern of growth in length of 48 individual boys and girls during the adolescent cycle of growth. Human Biology, 29: 83-122.

[2] Marubini, E., Resele, L. F., Tanner, J. M., \& Whitehouse, R. H (1972). The fit of Gompertz and Logistic curves to longitudinal data during adolescence on height, sitting height and biacromial diameter in boys and girls of the Harpenden Growth study. Human Biology, 44: 511-523.

[3] Preece, M. A., \& Baines, M. J. (1978). A new family of mathematical models describing the human growth curve. Annals of Human Biology, 5: 1-24.

[4] Largo, R. H., T. Gasser, A. Prader, W. Stuetzle and P. J. Huber. (1978). Analysis of the adolescent growth spurt using smoothing spline functions. Ann. Hum. Biol., 5: 421-434.

[5] Hauspie, R. C. (1989). Mathematical models for the study of individual growth patterns. Revue d'Epidémiologie et de Santé Publique, 37: 461-476.

[6] Fujii, K. (2017). Re-verification with regard to Scammon's growth curve proposal of Fujimmon's growth curve as a tentative Idea. American Journal of Sports Science, 5 (3): 14-20.

[7] Fujii, K. (2006). A scientific approach to growth and development- Physical information science for growth and development \& health-. Sankeisha, Nagoya. (in Japanese)

[8] Fujii, K., Mishima, T., Tanaka, N. (2021). Health Development Science in Body Information, Kyorinshoin, Tokyo. (in Japanese)

[9] Quetelet, A. (1835). Sur I' home et I' developpement de ses facultes. Essai sur physique sociale, Vol. 2, Bachelier.

[10] Keys, A., Fidanza, F., Karvonen, M. J., Kimura, N., and Taylor, HL. (1972). Indices of relative weight and obesity. J. Chronic. Dis., 25: 329-343. 
[11] Garrow, J. S., and Webster, J. (1985). Quetelet's index (W/H2) as a measure of fatness. Int. J. Obes. Relat. Metab. Disord., 9: 147-153.

[12] Wang, M. C., and Bachrach, L. K. (1996). Validity of the body mass index as an indicator of adiposity in an ethnically diverse population of youths. Am. J. Hum. Biol., 8: 641-651.

[13] Maynard, L. M., Wisemandle, W., Roche, A. F., Chumlea, W. C., Guo, S. S., and Siervogel, R. M. (2001). Childhood body composition in relation to body mass index. Pediatrics, 107: 344-350.

[14] Tahara, Y., Moji, K., Aoyagi, K., Nishizawa, S., Yukawa, K., Tsunawake, N., Muraki, S., and Mascie-Taylor, C. G. (2002). Age-related pattern of body density and body composition in Japanese males and females, 11 and 18 years of age. Am. J. Hum. Biol., 14: 327-337.

[15] Tanaka, N., Fujii, K., Asauchi, D., Ishigaki, T. (2007). Auxological approach to relational construction with regarding mutual relationship among body compositions, physical and motor fitness. Tokai Annual Report health and Physical Education, 29: 37-46. (in Japanese)

[16] Tanaka, N., Fujii, K., Ishigaki, T., Asauchi, D. (2008). An approach to evaluation chart of physical fitness based on difference of body form quality in junior high school boys. Tokai Annual Report health and Physical Education, 30: 33-42. (in Japanese)
[17] Tanaka, N., Fujii, K., Ishigaki, T., Nho, H. S., Kim S. H., Hanai, T. (2009). Auxological approach to relational construction among body compositions and physical fitness in Korean junior high school students. Japan Journal of Human Growth and Development Research, 41: 44-57. (in Japanese)

[18] Singh, D. (1994). Body fat distribution and perception of desirable female body shape by young black men and women. Int. J. Eat. Disord., 16: 289-294.

[19] Kuroki, T. (2007). Health • Aging - Life-span -Cultural record of Human and Life-. Chuokoronsya, Tokyo. (in Japanese)

[20] Fujii, K., Mishima, T., Watanabe, E., and Seki, K. (2010). Change with Age in Regression Construction of Fat Percentage for BMI in School-Age. Journal of Physiological Anthropology, 30: 69-76.

[21] Fujii, K., Tanaka, N., Ishigaki, T., Hanai, T., Kim, S. H., and Nho, H. (2008). Confirmation regarding Physical Fitness based on Polynomial Regression Evaluation of Body Fat Percentage for BMI in adolescence. The Korean Journal of Growth and Development, 16 (1): 43-47.

[22] Scammon, R. E., The measurement of the body in childhood. In Harris, J. A., Jackson, C. M., Paterson, D. G., and Scammon, R. E. (Eds) (1930). The Measurement of Man. Univ. of Minnesota Press, Minneapolis. 